\title{
Masers in G34.3+0.2: What more can 6.7-GHz Methanol masers tell us?
}

Jiyune Yi

Onsala Space Observatory, Chalmers University of Technology, 43992, Onsala, Sweden

Chris J. Phillips

Joint Institute for VLBI in Europe, Postbus 2, 7990 AA, Dwingeloo, The Netherlands

Roy S. Booth

Onsala Space Observatory, Chalmers University of Technology, 43992, Onsala, Sweden

\begin{abstract}
We present results of $6.7 \mathrm{GHz}$ methanol maser observations using the European VLBI Network (EVN) toward the G34.3+0.2 complex, which contains a prototypical cometary HII region and a dust enshrouded, probably emerging massive star, G34.24+0.13. We have investigated the methanol maser distribution in both objects and compared it with the $\mathrm{OH}$ and water masers in the main HII region. The masers in G34.24+0.13 lie on top of the $1.3 \mathrm{~mm}$ continuum emission showing that methanol masers are present, even at this early evolutionary stage.
\end{abstract}

\section{Introduction}

Interstellar $\mathrm{OH}$ and $\mathrm{H}_{2} \mathrm{O}$ masers in the Galaxy have been used as probes of Massive Star Formation (MSF) for more than three decades and during the last decade methanol masers have become an intensively used tracer of MSF. Together with interstellar maser sites, ultracompact HII (UCHII) regions, molecular outflows and jets, cool dust envelopes which radiate in the far-infrared, hot dust cocoons, bright in the near infrared, and hot molecular cores have all been suggested as signposts of MSF. Many observational studies of these manifestations of protostars have been presented. However, relatively few investigations have been able to make detailed physical connections between the signposts and the process of MSF. Such progress will come as more objects are observed in detail through high resolution multi-wavelength studies.

$\mathrm{OH}$ and $\mathrm{H}_{2} \mathrm{O}$ masers have been observed towards many northern sources together with emission from molecular lines with the comparable resolution. High resolution observations of methanol masers have been made only in in the last couple of years (Minier et al. 2001; Walsh et al. 1999). Some of these observations show that the methanol maser components are aligned with a linear velocity gradient along the line. This has been taken to indicate a 
rotating disc (Norris et al. 1998; Phillips et al. 1998). More observations at high resolution toward MSF regions are essential to study them in depth. With this in mind, we have made observations of the G34.26+0.15 HII complex. This HII complex consists of one prototypical cometary HII region, two UCHII regions located to the east of the cometary HII region, and a hot molecular core lying between them. The complex can be one of the best candidates to explore objects of MSF in different evolutionary stages, especially since the newly found G34.24+0.13MM is nearby, although isolated from the G34.26+0.15 complex and has only been detected at (sub)millimeter wavelength. In this paper we will discuss the methanol maser distribution to explore the kinematical properties of the sources.

\section{Observations}

VLBI observations of the methanol maser transition at $6.7 \mathrm{GHz}$ towards G34.26+0.15 and G34.24+0.13 were made on November 30, 1999 using the EVN. Two $2 \mathrm{MHz}$ bands centered on $V_{L S R}=58.5 \mathrm{~km} \mathrm{~s}^{-1}$ were divided into 1024 spectral channels, providing a spectral resolution of $0.18 \mathrm{~km} \mathrm{~s}^{-1}$ after Hanning smoothing. A line rest frequency of $6.6685192 \mathrm{GHz}$ was adopted for the $5_{1} \rightarrow$ $6_{0} A^{+}$transition. The data were calibrated using standard techniques. The synthesized beam was $\sim 8.6 \times 6.4$ mas.

\section{Results}

\subsection{G34.26+0.15}

The observed spectrum of the $\mathrm{CH}_{3} \mathrm{OH}$ maser is quite simple, covering a velocity range of only from 56.5 to $58.5 \mathrm{~km} \mathrm{~s}^{-1}$ (figure 2.2 , left). The VLBI observations
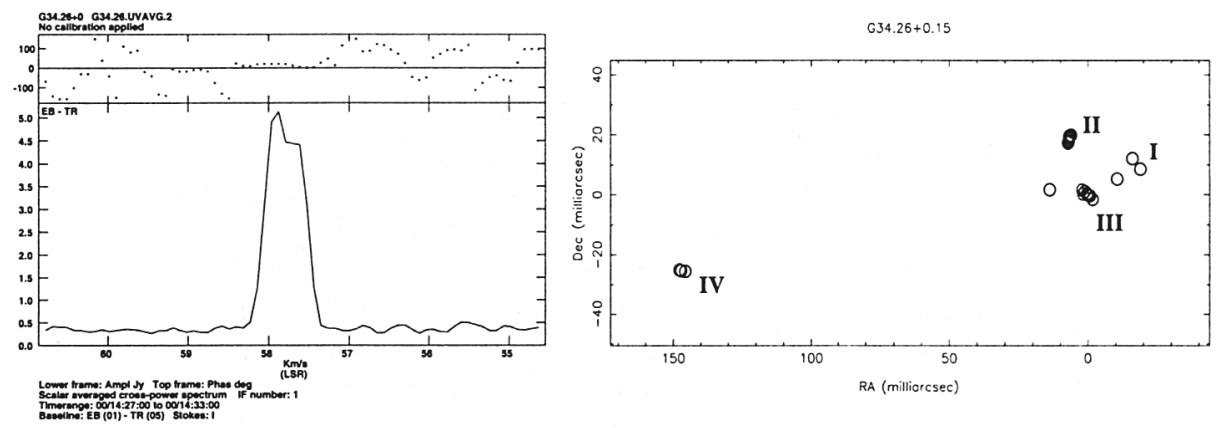

Figure 1. Cross-power spectrum (left) taken on Effelsberg-Torun baseline of the $6.7 \mathrm{GHz}$ maser emission from G34.26+0.15. VLBI map (right) shows four clusters of masers over the velocity range 56.6 to $58.5 \mathrm{~km} \mathrm{~s}^{-1}$

show that the spectral components are grouped into four clusters I, II, III and IV (figure 1, right); the cluster IV is separated by $\sim 160$ mas from the other three groups. Although the distributions of the maser spots are too simple to 
test a rotation disk hypothesis (Norris et al. 1998; Phillips 1998) we see a fair alignment of the maser spots showing a rough linear velocity gradient along a line extended over $\sim 170$ mas or $561 \mathrm{AU}$ at a distance of $3.3 \mathrm{kpc}$. We estimate an enclosed mass of $\sim 0.14 \mathrm{M}_{\odot}$ with the same methods as Norris et al. (1998) and include the value for interest. With an accuracy of $\sim 0.5 "$ (Ellingsen, private communication) the methanol emission is located on the south-west edge side of the compact component B (G34.26+0.15B) (Gaume, Fey \& Claussen 1994). On this map, the $\mathrm{OH}$ masers appear to be mostly located around the leading edge (head part) of the cometary component $\mathrm{C}$ and another isolated group associated with the UCHII B. Some of the $\mathrm{H}_{2} \mathrm{O}$ masers are found projected against the component $\mathrm{C}$ but most strong clusters of $\mathrm{H}_{2} \mathrm{O}$ masers are found about $2^{\prime \prime}$ east of the cometary component $\mathrm{C}$ where the ultracompact molecular core is located.

\subsection{G34.24+0.13}

The $350 \mu \mathrm{m}$ continuum source, G34.24+0.13MM was reported in 1998 (Hunter et al. 1998); it was not identified as a compact radio source in the $2 \mathrm{~cm}$ continuum map but the source is clearly detected at $225 \mathrm{GHz}$. The $6.7 \mathrm{GHz}$ methanol masers
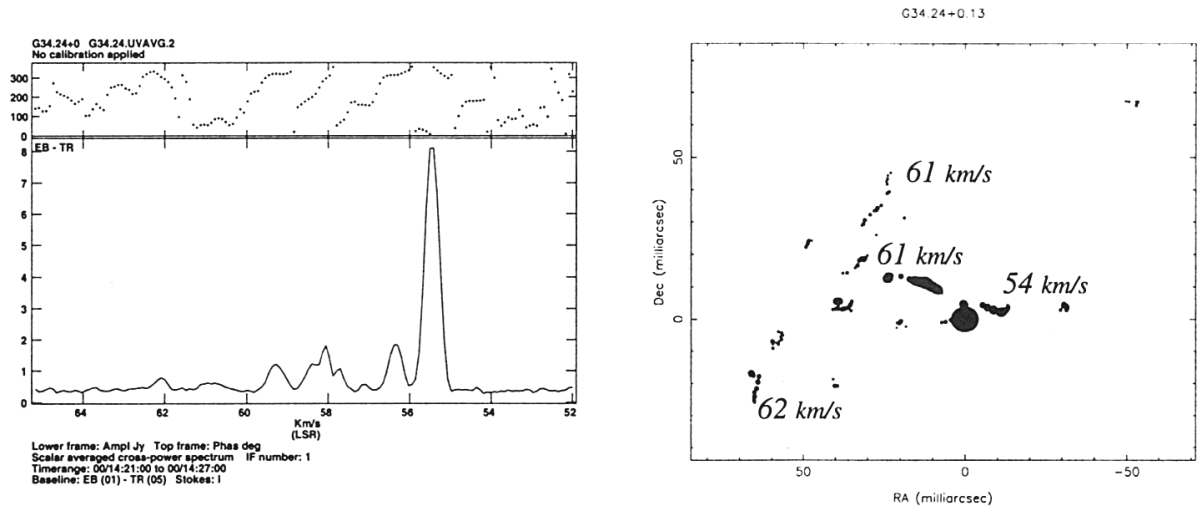

Figure 2. Cross-power spectrum (left) taken on Effelsberg-Torun baseline of the $6.7 \mathrm{GHz}$ maser emission from G34.24+0.13. VLBI map (right) shows complex distributions of masers over the velocity range 54 to $61 \mathrm{~km} \mathrm{~s}^{-1}$. The area of the solid circles is proportional to the flux density of the maser component

are near the center of the $225 \mathrm{GHz}$ continuum source. The spectrum of $6.7 \mathrm{GHz}$ masers (figure 2, left) is very rich in features and the VLBI map (figure 2, right) displays a complex distribution of masers. There is no simple linear distribution, however there is a line of masers roughly north-east with practically the same velocity $\left(\sim 61 \mathrm{~km} \mathrm{~s}^{-1}\right)$ and a rough east-west line of masers with a velocity gradient from 54 to $61 \mathrm{~km} \mathrm{~s}^{-1}$. There is another group of maser with a central velocity at about $55 \mathrm{~km} \mathrm{~s}^{-1}$, which when combined with the full distribution, form what might be described as a conical structure. Such structures might be related to outflows but our data is unable to qualify such a statement. 


\section{Discussions}

Methanol masers associated with G34.26+0.15 appear in two groups aligned approximately east-west. They are preferentially associated with the compact component $\mathrm{B}$ which also contains $\mathrm{OH}$ masers. There are no $\mathrm{H}_{2} \mathrm{O}$ masers associated with the UCHII B but most strong clusters of $\mathrm{H}_{2} \mathrm{O}$ masers are found about $2^{\prime \prime}$ east of the cometary component $\mathrm{C}$ where the ultracompact molecular core is located. If the positional coincidence between the strong clumps of water masers and the hot molecular clump is attributed to the extreme youth of the region, this region could be a good candidate for a hot molecular core on the verge of developing an UCHII region. It is situated on the east side of the head of the cometary HII region, which is presumably a more evolved site of on going massive star formation. We suggest that the association of methanol masers with the UCHII B is a good indication of a young UCHII region.

The complex distribution of methanol masers in G34.24+0.13 is rather exceptional for methanol masers. The estimated bolometric luminosity of G34.24+0.13MM ranges from 1600 to $6300 \mathrm{~L}_{\odot}$ corresponding to zero-age mainsequence stars B3 to B0 (Hunter et al. 1998). Osorio et al. (1999) derived a mass accretion rate and suggested that the energy source is too young to develop a detectable UCHII region. The presence of methanol masers in this source also supports the idea that methanol masers trace protostars.

\section{References}

Gaume, R.A., Fey, A.L., \& Claussen, M.J. 1994, ApJ, 432, 64

Hunter, T.R., Neugebauer, G., Benford, D.J., Matthews, K., Lis, D.C., Serabyn, E.,\& Phillips, T.G. 1998, ApJ, 493, L97

Minier, V., Conway, J.E., \& Booth, R.S. 2001, A\&A, 369, 278

Norris, R.P., Byleveld, S.E., Diamond, P.J., Ellingsen, S.P., Ferris, R.H., Gough, R.G., Kesteven, M.J., McCulloch, P.M., Phillips, C.J., Reynolds, J.E., Tzioumis, A.K., Takahashi, Y., Troup, E.R., \& Wellington, K.J. 1998, ApJ, 508, 275

Osorio, M., Lizano S., D'Alessio, P. 1999, ApJ, 525, 808

Phillips, C.J., Norris, R.P., Ellingsen, S.P., McCulloch, P.M. 1998, MNRAS, 300,1131

Walsh, A.J., Burton, M.G., Hyland, A.R., Robinson, G. 1999, MNRAS, 309, 905 